\title{
Stability in the Pseudo-state Formalism of Discrete Systems Controlled in the Presence of Intermittent Sensor Faults *
}

\author{
Dionísio, R.V. ${ }^{*, * *}$ Lemos, J.M. ${ }^{* *, * * *}$ \\ * ESTSetúbal/LabIM/Instituto Politécnico de Setúbal \\ Campus do IPS, Estefanilha, 2910-761 Setúbal, Portugal (e-mail: \\ rui.vilela@estsetubal.ips.pt). \\ ** Instituto Superior Técnico, Av. Rovisco Pais, 1049-001 Lisboa, \\ Portugal (e-mail: jlml@inesc-id.pt). \\ *** INESC-ID, R. Alves Redol 9, 1000-029 Lisboa, Portugal
}

\begin{abstract}
This paper presents sufficient conditions for the stabilization of open-loop unstable discrete time invariant systems, with nonlinear actuators, described in the pseudo-state formalism, stabilized by state feedback, when intermittent observations due to sensor faults occur. It is shown that the closed-loop system with feedback through a reconstructed signal, when, at least, one of the sensors is unavailable, remains uniformly exponentially stable, provided that the intervals of unavailability satisfy a certain time bound, even in the presence of state vanishing perturbations. The result is proved for a class of Hammerstein systems.
\end{abstract}

Keywords: Sensors' faults; Nonlinear actuators; Pseudo-state; Uniform exponential stability; Intermittent observations.

\section{INTRODUCTION}

In recent years the mass advent of digital communication networks and systems has boosted the integration of teleoperation in feedback control systems. Applications like unmanned vehicles Halberg et al. (1999) or Internet based real time control Overstreet (1999) provide significant examples raising, in turn, new problems.

This paper deals with one of such problems: if the communication channel through which feedback information passes is not completely reliable, sensors' measurements may not be available to the controller during some intervals of time. In such a situation, one has to couple the controller with a supervisor, which is able to discriminate between intervals of signal availability (availability time $T_{a_{i}}$ ) and unavailability (unavailability time $T_{u_{i+1}}$ ), and to generate an estimate of the plant's state during this $T_{u_{i+1}}$ intervals. Methods for detection and estimation for abruptly changing systems, Tugnait (1982), can be applied in the problem considered here. For that purpose an algorithm based on Bayesian decision could be implemented, for example.

Somehow related with the problem of temporary sensor unavailability presented in this paper is the problem of data packet dropout, and the problem of network-induced delay, in Networked Control Systems Zhang et al. (2001), and Estrada et al. (2006).

Biomedical applications provide as well, examples in that the sensor used for feedback is intermittently unavailable. In Lemos et al. (2005) the artifacts in the neuromuscular

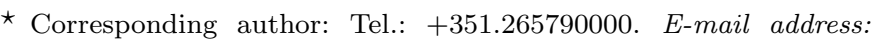
rui.vilela@estsetubal.ips.pt blockade level measurement in patients subject to general anesthesia are modeled as sensor faults. The occurrence of these faults is detected with a Bayesian algorithm and, during the periods of unavailability of the signal, the feedback controller is feed with an estimate generated by a model.

Also, the stability analysis for a real application example in the presence of intermittent faults is described in Gonzalez et al. (2001).

It is shown, throughout the paper, that with the above described scheme, the controlled open-loop unstable plant will be uniformly exponentially stable, if the time interval, during which at least one of the sensors' measure is unavailable, is somehow "small". Moreover, if the plant's state is perturbed by a class of vanishing perturbations, a similar stability result is derived.

The contributions of the paper consist in providing sufficient conditions for the stabilization of feedback controlled open-loop unstable systems with intermittent sensor faults, and with nonlinear actuators, described in the pseudo-state formalism.

The paper is organized in five sections and one Appendix. Section 2 makes a system description referring the functionality of the supervisor, and the way feedback system with nonlinear actuators behaves when intermittent sensor faults occur. Section 3 presents two new theorems with sufficient conditions for uniform exponential stability, for the system without vanishing perturbations, and for asymptotically stability, when vanishing perturbations are considered. Section 4 presents proof of nonexistence of necessary conditions for the afore theorems, and in section 


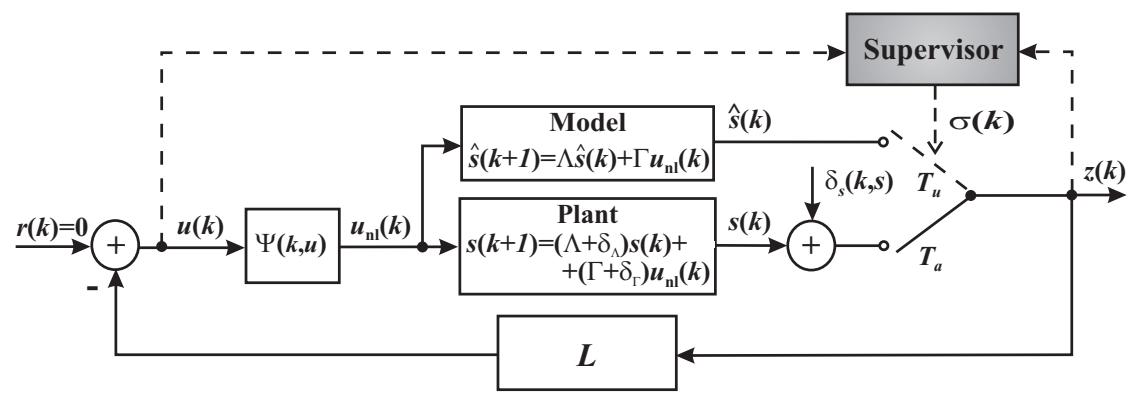

Fig. 1. Block diagram of a discrete feedback system with nonlinear actuator, and intermittent observations supervisor.

5 conclusions are drawn. Appendix gives a full proof of theorems.

\section{PROBLEM FORMULATION}

As to obtain a pseudo-state space description of a generic MIMO discrete plant, start with its I/O representation

$$
\begin{aligned}
& \operatorname{diag}\left(A_{1}\left(q^{-1}\right), A_{2}\left(q^{-1}\right), \ldots, A_{l}\left(q^{-1}\right)\right) \boldsymbol{y}(k)= \\
& =\left[\begin{array}{cccc}
B_{11}\left(q^{-1}\right) & B_{12}\left(q^{-1}\right) & \ldots & B_{1 p}\left(q^{-1}\right) \\
B_{21}\left(q^{-1}\right) & B_{22}\left(q^{-1}\right) & \cdots & B_{2 p}\left(q^{-1}\right) \\
\vdots & \vdots & \ddots & \vdots \\
B_{l 1}\left(q^{-1}\right) & B_{l 2}\left(q^{-1}\right) & \cdots & B_{l p}\left(q^{-1}\right)
\end{array}\right] \boldsymbol{u}(k)
\end{aligned}
$$

with $\boldsymbol{u} \in \mathbb{R}^{p}$ inputs, $\boldsymbol{y} \in \mathbb{R}^{l}$ outputs, and polynomials $A_{i}\left(q^{-1}\right)$, and $B_{i j}\left(q^{-1}\right)$ defined as

$$
\begin{array}{cc}
A_{i}\left(q^{-1}\right)=1+\sum_{k=1}^{n_{a}^{i}} a_{k}^{i} q^{-k} & B_{i j}\left(q^{-1}\right)=\sum_{k=1}^{n_{b}^{i j}} b_{k}^{i j} q^{-k} \\
n_{a}^{i}=\partial A_{i}\left(q^{-1}\right) & n_{b}^{i j}=\partial B_{i j}\left(q^{-1}\right)
\end{array}
$$

( $\partial$ is the degree of a polynomial, and $q^{-1}$ is the discrete time delay operator) subject to $a_{n_{a}^{i}}^{i} \cdot b_{n_{b}^{i j}}^{i j} \neq 0$, and $b_{1}^{i j} \neq 0$, $\forall j$, with $i=1, \ldots, l$, and $j=1, \ldots, p$. Equation (1) is re-written as

$$
\mathcal{A}\left(q^{-1}\right) \boldsymbol{y}(k)=\mathcal{B}\left(q^{-1}\right) \boldsymbol{u}(k)
$$

being $\mathcal{A}\left(q^{-1}\right)$, and $\mathcal{B}\left(q^{-1}\right)$ left co-primes. Vectors in equation (1), and equation (3) are defined as

$$
\begin{aligned}
\boldsymbol{u}(k) & :=\left[u_{1}(k) \ldots u_{p}(k)\right]^{T} \\
\boldsymbol{y}(k) & :=\left[y_{1}(k) \ldots y_{l}(k)\right]^{T}
\end{aligned}
$$

Introducing pseudo-state $\boldsymbol{s}(k)$

$$
\begin{aligned}
\boldsymbol{s}(k):= & {\left[y_{1}(k) \ldots y_{1}\left(k-n_{a}^{1}+1\right) \ldots\right.} \\
& \ldots y_{l}(k) \ldots y_{l}\left(k-n_{a}^{l}+1\right) \\
& u_{1}(k-1) \ldots u_{1}\left(k-n_{b}^{1}+1\right) \ldots \\
& \left.\ldots u_{p}(k-1) \ldots u_{p}\left(k-n_{b}^{p}+1\right)\right]^{T} \in \mathbb{R}^{n}
\end{aligned}
$$

where $n_{b}^{j}=\max \left\{n_{b}^{i j}: i=1, \ldots, l\right\}$, for each $j=1, \ldots, p$, and $n=n_{a}^{1}+\cdots+n_{a}^{l}+n_{b}^{1}+\cdots+n_{b}^{p}-p$. Notice that pseudo-state $\boldsymbol{s}(k)$ in equation (4) already has sensors' information at time $k$.

Finally, the discrete plant can be described by

$$
\left\{\begin{array}{l}
\boldsymbol{s}(k+1)=\Lambda \boldsymbol{s}(k)+\Gamma u(k) \\
y(k)=H \boldsymbol{s}(k)
\end{array}\right.
$$

For a description of matrices' $\Lambda, \Gamma$, and $H$ structure, see Mosca (1995).
Consider, now, the system depicted in Fig. 1, where the discrete plant, and model are described in the pseudo-state form, respectively, by

$$
\begin{aligned}
& \boldsymbol{s}(k+1)=\left(\Lambda+\delta_{\Lambda}\right) \boldsymbol{s}(k)+\left(\Gamma+\delta_{\Gamma}\right) \boldsymbol{u}_{\mathrm{nl}}(k) \\
& \hat{\boldsymbol{s}}(k+1)=\Lambda \hat{\boldsymbol{s}}(k)+\Gamma \boldsymbol{u}_{\mathrm{nl}}(k)
\end{aligned}
$$

with $s$ and $\hat{s} \in \mathbb{R}^{n}$, accessible for direct measurement (being $\hat{\boldsymbol{s}}$ the state estimate), $\boldsymbol{u}$ and $\boldsymbol{u}_{\mathrm{nl}} \in \mathbb{R}^{p}, \Lambda, \Gamma$, $\delta_{\Lambda}$, and $\delta_{\Gamma}$ are of appropriate dimensions, and $(\Lambda, \Gamma)$ is stabilizable. Moreover, $\delta_{\Lambda}$, and $\delta_{\Gamma}$ represent modeling uncertainties. It is assumed the plant is time invariant, and open-loop unstable.

The vector $\boldsymbol{u}_{\mathrm{nl}}$ represents the nonlinear input to both the plant and the model, $\boldsymbol{u}_{\mathrm{nl}}(k)=\boldsymbol{\Psi}(k, \boldsymbol{u})$. Memoryless nonlinearity, $\boldsymbol{\Psi}:[0, \infty) \times \mathbb{R}^{p} \rightarrow \mathbb{R}^{p}$, is said to satisfy a sector condition globally, Khalil (1996), if

$$
\left[\boldsymbol{\Psi}(k, \boldsymbol{u})-K_{\min } \boldsymbol{u}(k)\right]^{T}\left[\boldsymbol{\Psi}(k, \boldsymbol{u})-K_{\max } \boldsymbol{u}(k)\right] \leq 0
$$

$\forall t \geq 0, \forall \boldsymbol{u} \in \mathbb{R}^{p}$, for some real matrices $K_{\min }$ and $K_{\max }$, where $K=K_{\max }-K_{\min }$ is a positive definite symmetric matrix. The nonlinearity $\boldsymbol{\Psi}(k, \boldsymbol{u})$ is said to belong to a sector $\left[K_{\min }, K_{\max }\right]$.

Vector $\boldsymbol{\delta}_{\boldsymbol{s}}(k, \boldsymbol{s})$ represents the vanishing perturbations that affect the pseudo-state, and both the plant, and the model are assumed to be stabilized through a predictive controller, e.g., GPC, with control gains matrix $L$.

The supervisor is responsible for detection of sensors' measures interruptions, and for switching state feedback from plant to model (during unavailability time $T_{u}$ ), and from model to plant (during availability time $T_{a}$ ), through the switch controlling variable $\sigma$. An example of a supervisor based on Bayesian inference is provided in Lemos et al. (2003), and next briefly described.

One possibility for modeling sensors' measures interruptions is to consider that each observation (sensors' measures) $\boldsymbol{s}(k)$ (it is assumed that $\boldsymbol{s}_{\min } \leq \boldsymbol{s}(k) \leq \boldsymbol{s}_{\max }$ ), made at discrete time $k$, occurs under hypothesis $H_{0}$ with probability $p_{0}$, close to one, or under hypothesis $H_{1}$ with probability $\left(1-p_{0}\right)$, close to zero. Under hypothesis $H_{0}$ the observation is equal to the value of the state $\boldsymbol{s}(k)$, added by zero mean white Gaussian noise of (constant) variance $\sigma_{e}^{2}$. Under hypothesis $H_{1}$ a measure interruption occurs. In this case the observation is no longer related to the state $\boldsymbol{s}(k)$, but, instead, is given by a random variable $\eta(k)$ with a probability density function which is uniform in the range of measurement, from $s_{\min }$ to $\boldsymbol{s}_{\max }$.

According to a Bayesian approach, in order to detect that a given observation is actually noise, the probability 


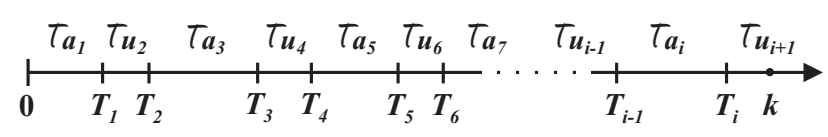

Fig. 2. Operation time line with availability intervals alternating with unavailability intervals.

of both hypothesis, given the observations, is computed. Next, they are compared, and if the probability associated with hypothesis $H_{1}$ is greater than the one associated with hypothesis $H_{0}$ it is decided that a measurement interruption has occurred. Under this decision, the observation is discarded and replaced by a forecast $\hat{\boldsymbol{s}}(k)$ of the true value of $\boldsymbol{s}(k)$, made from previous observations.

This yields a loss of performance with respect to the ideal situation in which the sensors are always available, and may pose stability problems if the plant is open-loop unstable.

In order to understand the system functioning, consider the time line of operation, depicted in Fig. 2, divided in alternate intervals where all sensors operate correctly $\left(T_{a_{j}}\right.$, with $\left.j=1,3,5,7, \ldots, i\right)$, and where, at least, one of them fails $\left(T_{u_{j}}\right.$, with $\left.j=2,4,6, \ldots, i-1, i+1\right)$ being replaced by the model estimate. Note that the index $j$ does not represent discrete instants of time, but is rather used to enumerate both the availability, $T_{a_{j}}$, and the unavailability, $T_{u_{j}}$, intervals. These intervals are identified in script font in the upper part of the time line of Fig. 2. The time instants corresponding to the beginning of each interval, whether it is an availability or an unavailability interval, are represented in the lower part of the time line of Fig. 2. Let $k_{0}$ denote the beginning of one such intervals. It is assumed that the first interval always corresponds to an availability interval, and that the intervals are open at their end. Furthermore, the time analysis always finishes in an unavailability interval at time $k$. Therefore, in a complete time sequence there are $(i+1)$ intervals, where $(i+1)$ is an even number.

The model initial sate $\hat{\boldsymbol{s}}$ is made equal to the last available observation of the state $s$ when an interrupted observation occurs $\left(\hat{\boldsymbol{s}}\left(k_{0}\right)=\boldsymbol{s}\left(k_{0}\right)=\boldsymbol{s}\left(k_{0}-1\right)\right)$, since the sate $\boldsymbol{s}$ is no longer available.

\section{SUFFICIENCY OF STABILITY CONDITIONS IN PSEUDO-STATE FORMALISM}

Regarding system's stability, consider the feedback system with nonlinear actuator function $\boldsymbol{\Psi}(k, \boldsymbol{u})$, and without the perturbation function $\boldsymbol{\delta}_{\boldsymbol{s}}(k, \boldsymbol{s})=0$, stabilized through a predictive controller, e.g., GPC, with control gains matrix $L$, see Fig. 1 . The reference signal, $r(k)$, is considered to be zero, $\forall k \geq 0$ (regulation problem).

Throughout the text, matrices norms are the ones induced by the Euclidean norm of vectors, being given by their largest singular value $\left(\|A\|=\sigma_{\max }[A]=\sigma_{A} \geq 0\right)$.

Proposition 1. Consider $K_{\min }=-\frac{\gamma_{2}}{2} I$ and $K_{\max }=\frac{\gamma_{2}}{2} I$, with $\gamma_{2}$ a finite positive constant. The nonlinearity $\boldsymbol{\Psi}(k, \boldsymbol{u})$ can be decomposed in a linear component and a nonlinear component

$$
\boldsymbol{\psi}_{\boldsymbol{s}}(k, \boldsymbol{u})=\boldsymbol{\Psi}(k, \boldsymbol{u})-K_{\min } \boldsymbol{u}(k)
$$

where $\boldsymbol{\psi}_{\boldsymbol{s}}(k, \boldsymbol{u})$ represents the nonlinear component, and verifies the sector condition

$$
\boldsymbol{\psi}_{\boldsymbol{s}}^{T}(k, \boldsymbol{u})\left[\boldsymbol{\psi}_{\boldsymbol{s}}(k, \boldsymbol{u})-K \boldsymbol{u}(k)\right] \leq 0
$$

Proof. The result is straightforward using equation (9) in equation (8), and considering matrix $K$ definition.

Proposition 2. For the defined matrices $K_{\min }$ and $K_{\max }$, the memoryless sector nonlinearity $\boldsymbol{\Psi}(k, \boldsymbol{u})$ is bounded by $\|\boldsymbol{\Psi}(k, \boldsymbol{u})\| \leq \frac{\gamma_{2}}{2}\|\boldsymbol{u}(k)\|, \forall t \geq 0, \forall \boldsymbol{u} \in \mathbb{R}^{p}$.

Proof. Replacing $K_{\min }$ and $K_{\max }$ by their respective values in equation $(8)$, and since $\boldsymbol{\Psi}^{T}(k, \boldsymbol{u}) \boldsymbol{u}(k)$ is a scalar, yields

$$
\|\boldsymbol{\Psi}(k, \boldsymbol{u})\|^{2}-\left(\frac{\gamma_{2}}{2}\right)^{2}\|\boldsymbol{u}(k)\|^{2} \leq 0
$$

By definition $\|\boldsymbol{\Psi}(k, \boldsymbol{u})\| \geq 0,\|\boldsymbol{u}(k)\| \geq 0$, and $\frac{\gamma_{2}}{2}>0$, which implies

$$
\|\boldsymbol{\Psi}(k, \boldsymbol{u})\| \leq \frac{\gamma_{2}}{2}\|\boldsymbol{u}(k)\|
$$

In order to find a bound on $\boldsymbol{\psi}_{\boldsymbol{s}}(k, \boldsymbol{u})$ starting from equation (9), using equation (10), and $K_{\min }$ definition, it follows that

$$
\left\|\boldsymbol{\psi}_{\boldsymbol{s}}(k, \boldsymbol{u})\right\| \leq \gamma_{2}\|\boldsymbol{u}(k)\|
$$

The state feedback of signal $\boldsymbol{z}(k)$

$$
\boldsymbol{u}(k)=-L \boldsymbol{z}(k)
$$

is implemented by $L$, a matrix of feedback gains assumed to stabilize the model. Furthermore, $\boldsymbol{z}(k)=\boldsymbol{s}(k)$ during availability intervals, when all sensors are working properly, and $\boldsymbol{z}(k)=\hat{\boldsymbol{s}}(k)$ during unavailability intervals, when measuring interruptions take place.

During availability intervals the plant state equation is

$$
\begin{aligned}
\boldsymbol{s}(k+1)= & {\left[\left(\Lambda+\delta_{\Lambda}\right)-\left(\Gamma+\delta_{\Gamma}\right) K_{\min } L\right] \boldsymbol{s}(k)+} \\
& +\left(\Gamma+\delta_{\Gamma}\right) \boldsymbol{\psi}_{\boldsymbol{s}}(-L \boldsymbol{s}(k))
\end{aligned}
$$

During unavailability intervals the plant state equation is

$$
\begin{aligned}
\boldsymbol{s}(k+1)= & \left(\Lambda+\delta_{\Lambda}\right) \boldsymbol{s}(k)+\left(\Gamma+\delta_{\Gamma}\right) . \\
& \cdot\left(\boldsymbol{\psi}_{\boldsymbol{s}}(-L \hat{\boldsymbol{s}}(k))-K_{\min } L \hat{\boldsymbol{s}}(k)\right)
\end{aligned}
$$

Define the plant closed-loop dynamics matrix as

$$
\bar{\Lambda}_{\delta_{\mathrm{CL}}}=\left(\Lambda+\delta_{\Lambda}\right)-\left(\Gamma+\delta_{\Gamma}\right) K_{\min } L=\Lambda_{\delta}-\Gamma_{\delta} K_{\min } L
$$

model closed-loop dynamics matrix as

$$
\bar{\Lambda}_{\mathrm{CL}}=\Lambda-\Gamma K_{\min } L
$$

plant open and closed-loop transition matrices

$$
\begin{aligned}
& \Phi_{\delta}\left(k, k_{0}\right)=\left(\Lambda+\delta_{\Lambda}\right)^{k-k_{0}}=\Lambda_{\delta}^{k-k_{0}} \\
& \bar{\Phi}_{\delta_{\mathrm{CL}}}\left(k, k_{0}\right)=\bar{\Lambda}_{\delta_{\mathrm{CL}}}^{k-k_{0}}
\end{aligned}
$$

model open and closed-loop transition matrices

$$
\begin{aligned}
& \Phi\left(k, k_{0}\right)=\Lambda^{k-k_{0}} \\
& \bar{\Phi}_{\mathrm{CL}}\left(k, k_{0}\right)=\bar{\Lambda}_{\mathrm{CL}}^{k-k_{0}}
\end{aligned}
$$

and matrix $P=K_{\min } L$.

Theorem 1. Consider the closed-loop system of Fig.1 where the model is open-loop unstable (bounded by $\left\|\Phi\left(k, k_{0}\right)\right\| \leq \alpha \beta^{k-k_{0}}$, with $\alpha \geq 1$, and $\beta>1$, finite constants), and that $\exists L$ stabilizing the closed-loop $\left(\left\|\bar{\Phi}_{\mathrm{CL}}\left(k, k_{0}\right)\right\| \leq \gamma \lambda^{k-k_{0}}\right.$, with $\gamma \geq 1$ a finite constant, and $0 \leq \lambda<1)$. The nonlinearity $\boldsymbol{\psi}_{\boldsymbol{s}}(k, \boldsymbol{u})$ satisfies $\left\|\boldsymbol{\psi}_{\boldsymbol{s}}(k, \boldsymbol{u})\right\| \leq \gamma_{2}\|\boldsymbol{u}(k)\|, \forall t \geq 0, \forall \boldsymbol{u} \in \mathbb{R}^{p}$. Consider, also, that $\sigma_{\Gamma}=\|\Gamma\|, \sigma_{L}=\|L\|, \sigma_{P}=\|P\|$ and that model uncertainties are bounded $\left\|\delta_{\Lambda}\right\| \leq \sigma_{\delta_{\Lambda}}$, and $\left\|\delta_{\Gamma}\right\| \leq \sigma_{\delta_{\Gamma}}$. The system with initial condition $\boldsymbol{s}(0)=\boldsymbol{s}_{0}$ is globally uniformly exponentially stable provided that the total 
unavailability time $T_{u}$, up to discrete time $k$ inside the unavailability interval $T_{u_{i+1}}$, satisfies

$$
\begin{aligned}
T_{u}< & \frac{\log M_{1}}{\log \left(\frac{\beta+\alpha \cdot \sigma_{\delta_{\Lambda}}}{N_{1}}\right)}-\frac{(i+1)}{2} . \\
& \cdot \frac{\log \left[\left(1+\frac{\left(\sigma_{\Gamma}+\sigma_{\delta_{\Gamma}}\right)\left(\sigma_{P}+\gamma_{2} \cdot \sigma_{L}\right) \gamma}{\left(\beta+\alpha \cdot \sigma_{\delta_{\Lambda}}\right)-\left(\lambda+\gamma \cdot \sigma_{\Gamma} \cdot \gamma_{2} \cdot \sigma_{L}\right)}\right) \alpha \gamma\right]}{\log \left(\frac{\beta+\alpha \cdot \sigma_{\delta_{\Lambda}}}{N_{1}}\right)}- \\
& -T_{a} \frac{\log \left(\frac{(\lambda+\gamma \Sigma)+\gamma\left(\sigma_{\Gamma}+\sigma_{\delta_{\Gamma}}\right) \gamma_{2} \cdot \sigma_{L}}{N_{1}}\right)}{\log \left(\frac{\beta+\alpha \cdot \sigma_{\delta_{\Lambda}}}{N_{1}}\right)}
\end{aligned}
$$

$M_{1} \geq 1$ is a finite constant, and $\Sigma=\sigma_{\delta_{\Lambda}}+\sigma_{\delta_{\Gamma}} . \sigma_{P}$ is such that verifies

$$
0 \leq \Sigma<\frac{1-\lambda}{\gamma}
$$

and $\gamma_{2}$ is the less of the following two inequalities

$$
\begin{aligned}
\gamma_{2}<\frac{1-(\lambda+\gamma \Sigma)}{\gamma\left(\sigma_{\Gamma}+\sigma_{\delta_{\Gamma}}\right) \sigma_{L}} \\
\gamma_{2}<\frac{\beta+\alpha \cdot \sigma_{\delta_{\Lambda}}-\lambda}{\gamma \cdot \sigma_{\Gamma} \cdot \sigma_{L}}
\end{aligned}
$$

and $0 \leq N_{1}<1$ is a constant constrained to

$$
N_{1}>(\lambda+\gamma \Sigma)+\gamma\left(\sigma_{\Gamma}+\sigma_{\delta_{\Gamma}}\right) \gamma_{2} . \sigma_{L}
$$

and $T_{a}$ is the total availability time.

Remark 1. Notice that since $\left(\beta+\alpha \cdot \sigma_{\delta_{\Lambda}}\right)>1$ then it must be $(\lambda+\gamma \Sigma)+\gamma\left(\sigma_{\Gamma}+\sigma_{\delta_{\Gamma}}\right) \gamma_{2} . \sigma_{L}<1$, which leads to equation (21), so that the bound on $T_{u}$ has a monotonous crescent linear relation with $T_{a}$ in the result from Theorem 1.

Remark 2. The constant $N_{1}$ represents an upper bound on the rate of exponential decay of the overall system. If $N_{1}<(\lambda+\gamma \Sigma)+\gamma\left(\sigma_{\Gamma}+\sigma_{\delta_{\Gamma}}\right) \gamma_{2} . \sigma_{L}$ then the result of Theorem 1 would indicate a negative solution for $T_{u}$, which, clearly, is not possible, since $T_{u} \in[0, \infty[$. Being $N_{1}>(\lambda+\gamma \Sigma)+\gamma\left(\sigma_{\Gamma}+\sigma_{\delta_{\Gamma}}\right) \gamma_{2} . \sigma_{L}$, then the bound on $T_{u}$ has also a monotonous crescent linear relation with $T_{a}$, as mentioned in the previous remark.

Remark 3. Constant $M_{1}$ represents an offset term for the upper bound function on the evolution of $\|\boldsymbol{s}(k)\|$. The bigger this constant is, the more conservative is the referred upper bound on uniform exponential stability.

Remark 4. Theorem 1 presents only conservative sufficient stability conditions for the system of Fig. 1.

A proof of Theorem 1 is presented in Appendix.

Next, consider the perturbation $\boldsymbol{\delta}_{\boldsymbol{s}}(k, \boldsymbol{s}) \neq 0$, such that $\boldsymbol{\delta}_{\boldsymbol{s}}:[0, \infty) \times D \rightarrow \mathbb{R}^{n}$ is piecewise continuous in $k$ and locally Lipschitz in $s$ on $[0, \infty) \times D$, and $D \subset \mathbb{R}^{n}$ is a domain that contains the origin $s=0$. Also, $\left\|\boldsymbol{\delta}_{\boldsymbol{s}}(k, s)\right\| \leq$ $\epsilon\|\boldsymbol{s}(k)\|, \forall k \geq 0, \forall \boldsymbol{s} \in D$, and $\epsilon$ is a nonnegative constant, meaning the perturbation satisfies a linear growth bound, therefore, considered a vanishing perturbation, Khalil (1996).

In availability intervals $T_{a_{j}}$, for $j=1,3,5, \ldots, i$, the system can be represented by the autonomous equation

$$
\boldsymbol{s}(k+1)=\boldsymbol{F}(k, \boldsymbol{s})
$$

where $\boldsymbol{F}(k, \boldsymbol{s})$, for the system depicted in Fig. 1, is

$$
\boldsymbol{F}(k, \boldsymbol{s})=\bar{\Lambda}_{\delta_{\mathrm{CL}}} \boldsymbol{s}(k)+\left(\Gamma+\delta_{\Gamma}\right) \cdot \boldsymbol{\psi}_{\boldsymbol{s}}(-L \boldsymbol{s}(k))
$$

with $k \in T_{a_{j}}$

Clearly, $\boldsymbol{F}(0)=0$ (from equation (8) and matrices' $K_{\min }$ and $K_{\max }$ definition in Proposition 1 , the sector memoryless nonlinearity verifies $\left.\boldsymbol{\psi}_{\boldsymbol{s}}(0)=0\right)$. Recalling the state equation (14) during unavailability intervals $T_{u_{j}}$, for $j=2,4,6, \ldots, i+1$, and the fact that the initial model state $\hat{\boldsymbol{s}}$ is made equal to the last available observation of the state $s$ when an interrupted observation occurs, $\left(\hat{\boldsymbol{s}}\left(k_{0}\right)=\boldsymbol{s}\left(k_{0}\right)=\boldsymbol{s}\left(k_{0}-1\right)\right)$, it is clearly understood that if the state becomes zero during an availability interval, then it will remain zero for all time instants belonging to any unavailability interval, that may occur. The function's $\boldsymbol{F}(k, \boldsymbol{s})$ branch related with the unavailability interval is not of obvious writing in terms only of $\boldsymbol{s}(k)$. It has an easier writing in terms of $\boldsymbol{s}(k)$, and of $\hat{\boldsymbol{s}}(k)$. Nevertheless, since these two states are related at the switching time between availability and unavailability intervals (as recalled above), it can be understood that during an unavailability interval $\boldsymbol{F}(k, \boldsymbol{s})$ exists.

It is important to stress out that an unavailability interval can not occur without having previously existed an availability interval. Bearing this in mind it is possible to state that $\boldsymbol{F}(0)=0, \forall k \geq 0$, (including availability, and unavailability intervals).

Also, the nonlinear system was proved to be globally uniformly exponentially stable, under conditions of Theorem 1, therefore, $\boldsymbol{F}(k, \boldsymbol{s})$ is Lipschitz not only near the origin, but in $\mathbb{R}^{n}$, verifying $\left\|\boldsymbol{F}\left(\boldsymbol{s}_{1}\right)-\boldsymbol{F}\left(\boldsymbol{s}_{2}\right)\right\| \leq L_{v}\left\|\boldsymbol{s}_{1}-\boldsymbol{s}_{2}\right\|$.

Combining the results from Theorem 1 with the above comments, and with the result presented in Scokaert et al. (1997), reproduced in the next theorem, leads to Theorem 3.

Theorem 2. Let $\boldsymbol{F}: \mathbb{R}^{n} \rightarrow \mathbb{R}^{n}$ satisfy a Lipschitz condition in a neighborhood of the origin, with $\boldsymbol{F}(0)=0$. If the origin is an exponentially stable fixed point of $\boldsymbol{s}(k+$ $1)=\boldsymbol{F}(k, \boldsymbol{s})$, it is an asymptotically stable fixed point of the perturbed system $\overline{\boldsymbol{s}}(k+1)=\boldsymbol{F}(k, \overline{\boldsymbol{s}})+\boldsymbol{\delta}_{\boldsymbol{s}}(k, \overline{\boldsymbol{s}})$.

Theorem 3. The non-perturbed system from Fig. $1, \boldsymbol{s}(k+$ $1)=\boldsymbol{F}(k, \boldsymbol{s})$, verifying Theorem 1 and Theorem 2 sufficient conditions, has a globally asymptotically stable fixed point of the perturbed system $\overline{\boldsymbol{s}}(k+1)=\boldsymbol{F}(k, \overline{\boldsymbol{s}})+\boldsymbol{\delta}_{\boldsymbol{s}}(k, \overline{\boldsymbol{s}})$ in the origin, and $\boldsymbol{\delta}_{\boldsymbol{s}}:[0, \infty) \times D \rightarrow \mathbb{R}^{n}$ is piecewise continuous in $k$ and locally Lipschitz in $s$ on $[0, \infty) \times D$, and $D \subset \mathbb{R}^{n}$ is a domain that contains the origin $s=0$. Also, $\left\|\boldsymbol{\delta}_{\boldsymbol{s}}(k, \boldsymbol{s})\right\| \leq \epsilon\|\boldsymbol{s}(k)\|, \forall k \geq 0, \forall \boldsymbol{s} \in D$, with $\epsilon$ a nonnegative constant, satisfies a linear growth bound.

Remark 5. This result is global since $\boldsymbol{F}(k, \boldsymbol{s})$ is Lipschitz continuous in $\mathbb{R}^{n}$, and the original system is uniformly exponentially stable, Scokaert et al. (1997).

\section{NONEXISTENCE OF NECESSARY CONDITIONS}

Since an intermittent unavailability occurrence of the information of, at least, one sensor is an unpredictable event in terms of its time of origin, and duration, it is not possible to determine the state norm $\boldsymbol{s}(k)$ supreme. Such, prevents Theorem 1 from being rewritten in a way that it could also reflect necessary conditions. This impossibility is justified by the fact that in availability intervals', the vector's $\boldsymbol{s}(k)$ state norm decays, and during unavailability intervals', the same norm, grows. Due to the unknown relative duration 
of the unavailability interval when compared with the availability interval, and the unknown time instant of unavailability interval occurrence, it is not deterministically possible to determine which value the vector's $\boldsymbol{s}(k)$ state norm will have in the beginning of the next availability interval. Therefore, it is not possible to write an exponential function with negative argument, candidate to supreme.

Regarding Theorem 1, as referred in Remark 3, constant $M_{1}$ represents an offset term for the superior limit function on the evolution of $\|\boldsymbol{s}(k)\|$. The bigger this constant is, the more conservative will be the afore mentioned superior limit initial value to the sufficient uniform exponential stability condition, rendering impossible the task of determining a minimum value for the constant $M_{1}$.

According to the previous arguments, it is only possible to obtain sufficient stability conditions and of conservative performance. However, if the referred unknowing about the intermittent observations, and if it is considered that they are unavailable at regular intervals of fixed known duration, then the problem gets simpler and can be put into the framework of another set of problems like data networks congestion, where communication is interrupted at predetermined instants and for fixed duration periods, until the network gets fluid, and data packages transmission is resumed. For this systems there are results similar to the ones here presented, but providing necessary and sufficient stability conditions Estrada et al. (2006).

\section{CONCLUSIONS}

The paper presents and proves sufficient conditions that allow a discrete time analysis of sensor unavailability (interrupted observations) intervals, bounding these intervals in order to state that the unstable open-loop plant represented in Fig.1, when controlled in closed-loop, is globally uniformly exponentially stable. These results are proved under the existence of modeling uncertainties and, if plant state vanishing perturbations occur, then global asymptotic stability is achieved for the perturbed system. The results were proved for systems with memoryless sector nonlinear actuators.

It is interesting to note that in a related work Zhang et al. (2001), a similar conservative theoretical result regarding uniform exponential stability is reported, showing that longer intervals of unavailability can be reached in practice and that these theoretical results might be too conservative for practical purposes.

\section{REFERENCES}

Estrada, T., H. Lin, P.J. Antsaklis (2006). Model-based control with intermittent feedback. 14th Mediterranean Conference on Control and Automation MED'06, Ancona, Italy, 28-30 June.

Gonzalez, O.R., W.S. Gray, A. Tejada, S. Patilkulkarni (2001). Stability analysis of upset recovery methods for electromagnetic interference. Proceedings of the 40th IEEE Conference on Decision and Control, Orlando, Florida USA, 4-7 December, vol. 5, pp. 4134-4139.

Halberg, E., I. Kaminer, A. Pascoal (1999). Development of a flight test system for unmanned air vehicle. IEEE Control Systems, 19(1), pp. 55-65.
Khalil, H.K. (1996). Nonlinear systems. Second edition, Prentice Hall, pp. 400-403.

Lemos, J.M., H. Magalhães, R.V. Dionísio, T. Mendonça (2003). Control of Physiological Variables in the Presence of Interrupted Feedback Measurements. 16th International Conference on Systems Engineering, Coventry, England, 9-11 September.

Lemos, J.M., H. Magalhães, T. Mendonça, R.V. Dionísio (2005). Control of Neuromuscular Blockade in the Presence of Sensor Faults. IEEE Transactions on Biomedical Engineering, 52(11), pp. 1902-1911.

Mosca, E. (1995). Optimal, predictive, and adaptive control. Prentice Hall, p. 86.

Overstreet, J.W., A. Tzes (1999). An Internet-based realtime control engineering laboratory. IEEE Control Systems, 19(5), pp. 19-34.

Rugh, W.J. (1996). Linear system theory. Second edition, Prentice Hall, pp. 452-455.

Scokaert, P.O.M., J.B. Rawlings, E.S. Meadows (1997). Discrete-time stability with perturbations: application to model predictive control. Automatica, 33(3), pp. 463470.

Tugnait, J. (1982). Detection and estimation for abruptly changing systems. Automatica, 18, pp. 607-615.

Zhang, Wei, Michael S. Branicky, Stephen M. Phillips (2001). Stability of networked control systems. IEEE Control Systems, 21(1), pp. 84-99.

\section{Appendix A}

Proof. (of Theorem 1) During availability time intervals $T_{a_{j}}$, with $j=1,3,5, \ldots, i$, is $\boldsymbol{z}(k)=\boldsymbol{s}(k)$, and the plant state $\boldsymbol{s}(k)$ evolves according to

$$
\begin{gathered}
\boldsymbol{s}(k)=\bar{\Phi}_{\delta_{\mathrm{CL}}}\left(k, k_{0}\right) \boldsymbol{s}\left(k_{0}\right)+\sum_{j=k_{0}}^{k-1} \bar{\Phi}_{\delta_{\mathrm{CL}}}(k, j+1) . \\
\cdot\left(\Gamma+\delta_{\Gamma}\right) \boldsymbol{\psi}_{\boldsymbol{s}}(-L \boldsymbol{s}(j)), \quad k \geq k_{0}+1
\end{gathered}
$$

During unavailability time intervals $T_{u_{j}}$, with $j=$ $2,4,6, \ldots, i-1, i+1$, is $\boldsymbol{z}(k)=\hat{\boldsymbol{s}}(k)$, the model initial sate $\hat{\boldsymbol{s}}$ is made equal to the last available observation of the state $s$ when an interrupted observation occurs $\left(\hat{\boldsymbol{s}}\left(k_{0}\right)=\boldsymbol{s}\left(k_{0}\right)=\boldsymbol{s}\left(k_{0}-1\right)\right)$, the model state $\hat{\boldsymbol{s}}(k)$ evolves according to

$$
\begin{gathered}
\hat{\boldsymbol{s}}(k)=\bar{\Phi}_{\mathrm{CL}}\left(k, k_{0}\right) \boldsymbol{s}\left(k_{0}\right)+\sum_{j=k_{0}}^{k-1} \bar{\Phi}_{\mathrm{CL}}(k, j+1) . \\
\cdot \Gamma \boldsymbol{\psi}_{\boldsymbol{s}}(-L \hat{\boldsymbol{s}}(j)), \quad k \geq k_{0}+1
\end{gathered}
$$

and the plant state $\boldsymbol{s}(k)$ evolves according to

$$
\begin{aligned}
\boldsymbol{s}(k)= & \Phi_{\delta}\left(k, k_{0}\right) \boldsymbol{s}\left(k_{0}\right)+\sum_{j=k_{0}}^{k-1} \Phi_{\delta}(k, j+1)\left(\Gamma+\delta_{\Gamma}\right) . \\
& \cdot\left[\boldsymbol{\psi}_{\boldsymbol{s}}(-L \hat{\boldsymbol{s}}(j))-P \hat{\boldsymbol{s}}(j)\right], \quad k \geq k_{0}+1
\end{aligned}
$$

It is assumed that the model is stabilized in closed-loop, and bounded by $\left\|\bar{\Phi}_{\mathrm{CL}}\left(k, k_{0}\right)\right\| \leq \gamma \lambda^{k-k_{0}}, k \geq k_{0}$, with $0 \leq \lambda<1$ and $\gamma \geq 1$, and that the model is open-loop unstable, but bounded by $\left\|\Phi\left(k, k_{0}\right)\right\| \leq \alpha \beta^{k-k_{0}}, k \geq k_{0}$, with $\beta>1$ and $\alpha \geq 1$.

For bounded model uncertainties $\left\|\delta_{\Lambda}\right\| \leq \sigma_{\delta_{\Lambda}}$, and considering the bound on $\left\|\Phi\left(k, k_{0}\right)\right\|$, with $\bar{\beta}>1$ (this corresponds to assume an unfavorable situation), it can be 
proved, through the use of a Gronwall-Bellman type of inequality for sequences, as presented in Rugh (1996), if $\delta_{\Lambda}$ is seen as a perturbation in the system $\boldsymbol{s}(k+1)=$ $\left(\Lambda+\delta_{\Lambda}\right) s(k)$, that $\left\|\Phi_{\delta}\left(k, k_{0}\right)\right\| \leq \alpha\left(\beta+\alpha \cdot \sigma_{\delta_{\Lambda}}\right)^{k-k_{0}}$, with $\left(\beta+\alpha \cdot \sigma_{\delta_{\Lambda}}\right)>1$. This means, as expected, that if the model dynamics are open-loop unstable, then there will be a $\delta_{A}$ such that the plant dynamics will be open-loop unstable (the use of a continuity argumentation could also explain such assertion). A similar proof can be given for the stability of the plant in closed-loop since the model is stable in closed-loop $\left(\left\|\bar{\Phi}_{\mathrm{CL}}\left(k, k_{0}\right)\right\| \leq \gamma \lambda^{k-k_{0}}\right.$, with $0 \leq \lambda<1)$. Recurring, again, to the referred Gronwall-Bellman type of inequality for sequences, and considering that $\left(\delta_{\Lambda}-\delta_{\Gamma} P\right)$ is seen as a perturbation in the system $\boldsymbol{s}(k+1)=\left[\left(\Lambda+\delta_{\Lambda}\right)-\left(\Gamma+\delta_{\Gamma}\right) P\right] \boldsymbol{s}(k)$, it can be proved that $\left\|\bar{\Phi}_{\delta_{\mathrm{CL}}}\left(k, k_{0}\right)\right\| \leq \gamma(\lambda+\gamma \Sigma)^{k-k_{0}}, k \geq k_{0}$, with $0 \leq \Sigma<\frac{1-\lambda}{\gamma}$, and $\Sigma=\sigma_{\delta_{\Lambda}}+\sigma_{\delta_{\Gamma}} . \sigma_{P}$, Rugh (1996).

Upper bounds for equation (A.1) during availability time intervals, and for equation (A.3) during unavailability time intervals, are obtained, respectively

$$
\begin{aligned}
\|\boldsymbol{s}(k)\|= & \| \bar{\Phi}_{\delta_{\mathrm{CL}}}\left(k, k_{0}\right) \boldsymbol{s}\left(k_{0}\right)+\sum_{j=k_{0}}^{k-1} \bar{\Phi}_{\delta_{\mathrm{CL}}}(k, j+1) . \\
& \cdot\left(\Gamma+\delta_{\Gamma}\right) \boldsymbol{\psi}_{\boldsymbol{s}}(-L \boldsymbol{s}(j)) \| \\
\|\boldsymbol{s}(k)\|= & \| \Phi_{\delta}\left(k, k_{0}\right) \boldsymbol{s}\left(k_{0}\right)+\sum_{j=k_{0}}^{k-1} \Phi_{\delta}(k, j+1) \\
& \cdot\left(\Gamma+\delta_{\Gamma}\right)\left[\boldsymbol{\psi}_{\boldsymbol{s}}(-L \hat{\boldsymbol{s}}(j))-P \hat{\boldsymbol{s}}(j)\right] \|
\end{aligned}
$$

From equation (A.4) and equation (A.5), recalling that $\|\Gamma\|=\sigma_{\Gamma},\left\|\delta_{\Gamma}\right\| \leq \sigma_{\delta_{\Gamma}},\|L\|=\sigma_{L}$, and considering equation (11), yields, respectively

$$
\begin{aligned}
\|\boldsymbol{s}(k)\| \leq & \gamma(\lambda+\gamma \Sigma)^{k-k_{0}} \cdot\left\|\boldsymbol{s}\left(k_{0}\right)\right\|+\sum_{j=k_{0}}^{k-1} \gamma(\lambda+\gamma \Sigma)^{k-j-1} . \\
\|\boldsymbol{s}(k)\| \leq & \alpha\left(\beta+\alpha \cdot \sigma_{\delta_{\Lambda}}\right)^{k-k_{0}} \cdot\left\|\boldsymbol{s}\left(k_{0}\right)\right\|+ \\
+ & \sum_{j=k_{0}}^{k-1} \alpha\left(\beta+\alpha . \sigma_{\delta_{\Lambda}}\right)^{k-j-1} . \\
& \cdot\left(\sigma_{\Gamma}+\sigma_{\delta_{\Gamma}}\right)\left(\sigma_{P}+\gamma_{2} . \sigma_{L}\right) \cdot\|\hat{\boldsymbol{s}}(j)\|
\end{aligned}
$$

Applying the Gronwall-Bellman type of inequality for sequences to equation (A.6), gives

$$
\|\boldsymbol{s}(k)\| \leq \gamma\left[(\lambda+\gamma \Sigma)+\gamma\left(\sigma_{\Gamma}+\sigma_{\delta_{\Gamma}}\right) \gamma_{2} \cdot \sigma_{L}\right]^{k-k_{0}} \cdot\left\|\boldsymbol{s}\left(k_{0}\right)\right\|
$$

Consider now, the upper bound for equation (A.2) obtained from

$$
\|\hat{\boldsymbol{s}}(k)\| \leq \gamma \lambda^{k-k_{0}} \cdot\left\|\boldsymbol{s}\left(k_{0}\right)\right\|+\sum_{j=k_{0}}^{k-1} \gamma \lambda^{k-j-1} \sigma_{\Gamma} \cdot \gamma_{2} \cdot \sigma_{L} \cdot\|\hat{\boldsymbol{s}}(j)\|
$$

Making use, once again, of the Gronwall-Bellman type of inequality for sequences, and recalling that $\hat{\boldsymbol{s}}\left(k_{0}\right)=$ $\boldsymbol{s}\left(k_{0}\right)=\boldsymbol{s}\left(k_{0}-1\right)$, yields

$$
\|\hat{\boldsymbol{s}}(k)\| \leq \gamma\left(\lambda+\gamma \cdot \sigma_{\Gamma} \cdot \gamma_{2} \cdot \sigma_{L}\right)^{k-k_{0}} \cdot\left\|\boldsymbol{s}\left(k_{0}\right)\right\|
$$

Replacing equation (A.10) in equation (A.7)

$$
\begin{aligned}
\|s(k)\| \leq & {\left[\alpha\left(\beta+\alpha \cdot \sigma_{\delta_{\Lambda}}\right)^{k-k_{0}}+\alpha\left(\sigma_{\Gamma}+\sigma_{\delta_{\Gamma}}\right)\left(\sigma_{P}+\gamma_{2} \cdot \sigma_{L}\right) \gamma \cdot\right.} \\
& \cdot\left(\beta+\alpha \cdot \sigma_{\delta_{\Lambda}}\right)^{k-1}\left(\lambda+\gamma \cdot \sigma_{\Gamma} \cdot \gamma_{2} \cdot \sigma_{L}\right)^{-k_{0}} \cdot \quad(\mathrm{A} .11) \\
& \left.\cdot \sum_{j=k_{0}}^{k-1}\left(\frac{\lambda+\gamma \cdot \sigma_{\Gamma} \cdot \gamma_{2} \cdot \sigma_{L}}{\beta+\alpha \cdot \sigma_{\delta_{\Lambda}}}\right)^{j}\right] \cdot\left\|\boldsymbol{s}\left(k_{0}\right)\right\|
\end{aligned}
$$

Consider, now, the sum of the $\left(k-k_{0}\right)$ terms of a geometric progression with ratio $r$

$$
\sum_{j=k_{0}}^{k-1} r^{j}=\frac{r^{k_{0}}-r^{k}}{1-r}
$$

If $|r|<1$, then, as $k \rightarrow \infty$, equation (A.12) becomes

$$
\sum_{j=k_{0}}^{k-1} r^{j}=\frac{r^{k_{0}}}{1-r}
$$

Using equation (A.12) in equation (A.11), gives

$$
\begin{aligned}
\|\boldsymbol{s}(k)\| \leq & {\left[\alpha\left(\beta+\alpha \cdot \sigma_{\delta_{\Lambda}}\right)^{k-k_{0}}+\alpha\left(\sigma_{\Gamma}+\sigma_{\delta_{\Gamma}}\right)\left(\sigma_{P}+\gamma_{2} \cdot \sigma_{L}\right) \gamma \cdot\right.} \\
& \left.\cdot \frac{\left(\beta+\alpha \cdot \sigma_{\delta_{\Lambda}}\right)^{k-k_{0}}-\left(\lambda+\gamma \cdot \sigma_{\Gamma} \cdot \gamma_{2} \cdot \sigma_{L}\right)^{k-k_{0}}}{\left(\beta+\alpha \cdot \sigma_{\delta_{\Lambda}}\right)-\left(\lambda+\gamma \cdot \sigma_{\Gamma} \cdot \gamma_{2} \cdot \sigma_{L}\right)}\right] . \\
& \cdot\left\|\boldsymbol{s}\left(k_{0}\right)\right\|
\end{aligned}
$$

Providing $\gamma_{2}<\frac{\beta+\alpha . \sigma_{\delta_{\Lambda}}-\lambda}{\gamma \cdot \sigma_{\Gamma} \cdot \sigma_{L}}$, and considering equation (A.13), after some calculations, equation (A.14) yields

$$
\begin{aligned}
\|\boldsymbol{s}(k)\| \leq & {\left[1+\frac{\left(\sigma_{\Gamma}+\sigma_{\delta_{\Gamma}}\right) \cdot\left(\sigma_{P}+\gamma_{2} \cdot \sigma_{L}\right) \gamma}{\left(\beta+\alpha . \sigma_{\delta_{\Lambda}}\right)-\left(\lambda+\gamma . \sigma_{\Gamma} \cdot \gamma_{2} . \sigma_{L}\right)}\right] . } \\
& \cdot \alpha\left(\beta+\alpha . \sigma_{\delta_{\Lambda}}\right)^{k-k_{0}} \cdot\left\|\boldsymbol{s}\left(k_{0}\right)\right\|
\end{aligned}
$$

The complete state evolution from time instant $k=0$, up to the final time instant at $k \in T_{u_{i+1}}$ is given by the alternate product of equations (A.1) and (A.3), where $\hat{\boldsymbol{s}}\left(k_{0}\right)=\boldsymbol{s}\left(k_{0}\right)=\boldsymbol{s}\left(k_{0}-1\right)$ is considered. Applying the results from equations (A.8) and (A.15) to this product

$$
\begin{aligned}
\|\boldsymbol{s}(k)\| \leq & c_{1}^{\frac{i+1}{2}}\left(\beta+\alpha . \sigma_{\delta_{\Lambda}}\right)^{T_{u}} . \\
& \cdot\left[(\lambda+\gamma \Sigma)+\gamma\left(\sigma_{\Gamma}+\sigma_{\delta_{\Gamma}}\right) \gamma_{2} . \sigma_{L}\right]^{T_{a}} \cdot\left\|\boldsymbol{s}_{0}\right\|
\end{aligned}
$$

where $T_{u}$ and $T_{a}$ represent the entire duration of all unavailability and availability time intervals, respectively, and

$$
c_{1}:=\left[1+\frac{\left(\sigma_{\Gamma}+\sigma_{\delta_{\Gamma}}\right) \cdot\left(\sigma_{P}+\gamma_{2} \cdot \sigma_{L}\right) \gamma}{\left(\beta+\alpha . \sigma_{\delta_{\Lambda}}\right)-\left(\lambda+\gamma \cdot \sigma_{\Gamma} \cdot \gamma_{2} \cdot \sigma_{L}\right)}\right] \alpha \gamma
$$

For the system to be uniformly exponentially stable, it must verify $\|s(k)\| \leq M_{1} N_{1}^{k-k_{0}}\left\|s\left(k_{0}\right)\right\|, k \geq k_{0}$, with $M_{1} \geq 1$, and $0 \leq N_{1}<1$. Therefore, from equation (A.16), and considering $k_{0}=0$, and $\left(k-k_{0}\right)=T_{u}+T_{a}$

$$
\begin{gathered}
c_{1}^{\frac{i+1}{2}}\left(\beta+\alpha . \sigma_{\delta_{\Lambda}}\right)^{T_{u}}\left[(\lambda+\gamma \Sigma)+\gamma\left(\sigma_{\Gamma}+\sigma_{\delta_{\Gamma}}\right) \gamma_{2} . \sigma_{L}\right]^{T_{a}} \leq \\
\leq M_{1} N_{1}^{T_{u}+T_{a}} \Rightarrow T_{u} \leq \\
\leq \frac{\log M_{1}-\frac{i+1}{2} \log c_{1}-T_{a} \log \left(\frac{\left[(\lambda+\gamma \Sigma)+\gamma\left(\sigma_{\Gamma}+\sigma_{\delta_{\Gamma}}\right) \gamma_{2} . \sigma_{L}\right]}{N_{1}}\right)}{\log \left(\frac{\beta+\alpha \cdot \sigma_{\delta_{\Lambda}}}{N_{1}}\right)}
\end{gathered}
$$

Replacing equation (A.17) in equation (A.18) gives the desired result from Theorem 1 subject to the constraints $\Sigma<\frac{1-\lambda}{\gamma}$, and $\gamma_{2}<\frac{\beta+\alpha \cdot \sigma_{\delta_{\Lambda}}-\lambda}{\gamma \cdot \sigma_{\Gamma} \cdot \sigma_{L}}$. The result holds globally since it is valid for any $\left\|\boldsymbol{s}\left(k_{0}\right)\right\|$. 